\title{
Article \\ Some Bounds for the Complex Čebyšev Functional of Functions of Bounded Variation
}

\author{
Silvestru Sever Dragomir 1,2 (D)
}

Citation: Dragomir, S.S. Some Bounds for the Complex Čebyšev Functional of Functions of Bounded Variation. Symmetry 2021, 13, 990. https://doi.org/10.3390/sym13060990

Academic Editors: Alina Alb Lupas and Roman Ger

Received: 27 April 2021

Accepted: 24 May 2021

Published: 2 June 2021

Publisher's Note: MDPI stays neutral with regard to jurisdictional claims in published maps and institutional affiliations.

\section{Copyright: (C) 2021 by the authors.} Licensee MDPI, Basel, Switzerland. This article is an open access article distributed under the terms and conditions of the Creative Commons Attribution (CC BY) license (https:// creativecommons.org/licenses/by/ $4.0 /)$.
1 Mathematics, College of Engineering \& Science Victoria University, P.O. Box 14428, Melbourne City 8001, Australia; sever.dragomir@vu.edu.au

2 DST-NRF Centre of Excellence in the Mathematical and Statistical Sciences, School of Computer Science \& Applied Mathematics, University of the Witwatersrand, Private Bag 3, Johannesburg 2050, South Africa

Abstract: In this paper, we provide several bounds for the modulus of the complex Čebyšev functional. Applications to the trapezoid and mid-point inequalities, that are symmetric inequalities, are also provided.

Keywords: measurable functions; integrable functions; functions of bounded variation; CauchyBunyakovsky-Schwarz inequality; Grüss' type inequalities; Čebyšev functional

\section{Introduction}

For Lebesgue integrable functions $f, g:[a, b] \rightarrow \mathbb{C}$ we consider the complex Čebyšev functional

$$
C(f, g):=\frac{1}{b-a} \int_{a}^{b} f(t) g(t) d t-\frac{1}{b-a} \int_{a}^{b} f(t) d t \frac{1}{b-a} \int_{a}^{b} g(t) d t
$$

For two integrable real-valued functions $f, g:[a, b] \rightarrow \mathbb{R}$, in order to compare the integral mean of the product with the product of the integral means, in 1934, G. Grüss [1] showed that

$$
|C(f, g)| \leq \frac{1}{4}(M-m)(N-n)
$$

provided $m, M, n, N$ are real numbers with the property that

$$
-\infty<m \leq f \leq M<\infty, \quad-\infty<n \leq g \leq N<\infty \text { a.e. on }[a, b] .
$$

The constant $\frac{1}{4}$ is most possible in (1) in the sense that it cannot be replaced by a smaller one. For other results, see [2-7].

To extend this symmetric inequality for complex-valued functions we need the following preparations.

For $\phi, \Phi \in \mathbb{C}$ and $[a, b]$ an interval of real numbers, define the sets of complex-valued functions (see $[4,8,9]$ )

$$
\bar{U}_{[a, b]}(\phi, \Phi):=\{g:[a, b] \rightarrow \mathbb{C} \mid \operatorname{Re}[(\Phi-g(t))(\overline{g(t)}-\bar{\phi})] \geq 0 \text { for a.e. } t \in[a, b]\}
$$

and

$$
\bar{\Delta}_{[a, b]}(\phi, \Phi):=\left\{g:[a, b] \rightarrow \mathbb{C}|| g(t)-\frac{\phi+\Phi}{2}\left|\leq \frac{1}{2}\right| \Phi-\phi \mid \text { for a.e. } t \in[a, b]\right\} .
$$

For any $\phi, \Phi \in \mathbb{C}, \phi \neq \Phi$, we have that $\bar{U}_{[a, b]}(\phi, \Phi)$ and $\bar{\Delta}_{[a, b]}(\phi, \Phi)$ are nonempty, convex and closed sets and

$$
\bar{U}_{[a, b]}(\phi, \Phi)=\bar{\Delta}_{[a, b]}(\phi, \Phi)
$$


We observe that for any $z \in \mathbb{C}$ we have the equivalence

$$
\left|z-\frac{\phi+\Phi}{2}\right| \leq \frac{1}{2}|\Phi-\phi|
$$

if and only if

$$
\operatorname{Re}[(\Phi-z)(\bar{z}-\bar{\phi})] \geq 0
$$

This follows from the equality

$$
\frac{1}{4}|\Phi-\phi|^{2}-\left|z-\frac{\phi+\Phi}{2}\right|^{2}=\operatorname{Re}[(\Phi-z)(\bar{z}-\bar{\phi})]
$$

that holds for any $z \in \mathbb{C}$.

The equality (3) is thus a simple consequence of this fact.

For any $\phi, \Phi \in \mathbb{C}, \phi \neq \Phi$,we also have that

$$
\begin{aligned}
\bar{U}_{[a, b]}(\phi, \Phi)= & \{g:[a, b] \rightarrow \mathbb{C} \mid(\operatorname{Re} \Phi-\operatorname{Re} g(t))(\operatorname{Re} g(t)-\operatorname{Re} \phi) \\
& +(\operatorname{Im} \Phi-\operatorname{Im} g(t))(\operatorname{Im} g(t)-\operatorname{Im} \phi) \geq 0 \text { for a.e. } t \in[a, b]\} .
\end{aligned}
$$

Now, if we assume that $\operatorname{Re}(\Phi) \geq \operatorname{Re}(\phi)$ and $\operatorname{Im}(\Phi) \geq \operatorname{Im}(\phi)$, then we can define the following set of functions as well:

$$
\begin{aligned}
\bar{S}_{[a, b]}(\phi, \Phi) & :=\{g:[a, b] \rightarrow \mathbb{C} \mid \operatorname{Re}(\Phi) \geq \operatorname{Re} g(t) \geq \operatorname{Re}(\phi) \\
& \text { and } \operatorname{Im}(\Phi) \geq \operatorname{Im} g(t) \geq \operatorname{Im}(\phi) \text { for a.e. } t \in[a, b]\} .
\end{aligned}
$$

One can easily observe that $\bar{S}_{[a, b]}(\phi, \Phi)$ is closed, convex and

$$
\varnothing \neq \bar{S}_{[a, b]}(\phi, \Phi) \subseteq \bar{U}_{[a, b]}(\phi, \Phi)
$$

This fact provides also numerous example of complex functions belonging to the class $\bar{\Delta}_{[a, b]}(\phi, \Phi)$.

In [4] we obtained the following complex version of Grüss' inequality:

$$
|C(f, \bar{g})| \leq \frac{1}{4}|\Phi-\phi||\Psi-\psi|
$$

provided $f \in \bar{\Delta}_{[a, b]}(\phi, \Phi)$ and $g \in \bar{\Delta}_{[a, b]}(\psi, \Psi)$, where $\bar{g}$ denotes the complex conjugate function of $g$.

We denote the variance of the complex-valued function $f:[a, b] \rightarrow \mathbb{C}$ by $D(f)$ and defined as

$$
D(f)=[C(f, \bar{f})]^{1 / 2}=\left[\frac{1}{b-a} \int_{a}^{b}|f(t)|^{2} d t-\left|\frac{1}{b-a} \int_{a}^{b} f(t) d t\right|^{2}\right]^{1 / 2},
$$

where $\bar{f}$ denotes the complex conjugate function of $f$.

If we apply the inequality (7) for $g=f$, then we obtain

$$
D(f) \leq \frac{1}{2}|\Phi-\phi|
$$

We observe that, if $g \in \bar{\Delta}_{[a, b]}(\psi, \Psi)$, then $\left|g(t)-\frac{\psi+\Psi}{2}\right| \leq \frac{1}{2}|\Psi-\psi|$ for a.e. $t \in[a, b]$ that is equivalent to $\left|\overline{g(t)}-\frac{\bar{\psi}+\bar{\Psi}}{2}\right| \leq \frac{1}{2}|\bar{\Psi}-\bar{\psi}|$ meaning that $\bar{g} \in \bar{\Delta}_{[a, b]}(\bar{\psi}, \bar{\Psi})$ and by (7), for $\bar{g}$ instead of $g$ we also have

$$
|C(f, g)| \leq \frac{1}{4}|\Phi-\phi||\Psi-\psi|
$$


provided $f \in \bar{\Delta}_{[a, b]}(\phi, \Phi)$ and $g \in \bar{\Delta}_{[a, b]}(\psi, \Psi)$.

We can also consider the following quantity associated with a complex-valued function $f:[a, b] \rightarrow \mathbb{C}$,

$$
E(f):=|C(f, f)|^{1 / 2}=\left|\frac{1}{b-a} \int_{a}^{b} f^{2}(t) d t-\left(\frac{1}{b-a} \int_{a}^{b} f(t) d t\right)^{2}\right|^{1 / 2} .
$$

Using (9) we also have

$$
E(f) \leq \frac{1}{2}|\Phi-\phi|
$$

For an integrable function $f:[a, b] \rightarrow \mathbb{C}$, consider the mean deviation of $f$ defined by

$$
R(f):=\frac{1}{b-a} \int_{a}^{b}\left|f(t)-\frac{1}{b-a} \int_{a}^{b} f(s) d s\right| d t .
$$

The following result holds (see [10] or the more extensive preprint version [11]).

Theorem 1. Let $f:[a, b] \rightarrow \mathbb{C}$ be of bounded variation on $[a, b]$ and $g:[a, b] \rightarrow \mathbb{C}$ a Lebesgue integrable function on $[a, b]$. Then

$$
|C(f, g)| \leq \frac{1}{2} \bigvee_{a}^{b}(f) R(g) \leq \frac{1}{2} \bigvee_{a}^{b}(f) D(g)
$$

where $\bigvee_{a}^{b}(f)$ denotes the total variation of $f$ on the interval $[a, b]$. The constant $\frac{1}{2}$ is best possible in (11).

Corollary 1. If $f, g:[a, b] \rightarrow \mathbb{C}$ are of bounded variation on $[a, b]$, then

$$
|C(f, g)| \leq \frac{1}{2} \bigvee_{a}^{b}(f) R(g) \leq \frac{1}{2} \bigvee_{a}^{b}(f) D(g) \leq \frac{1}{4} \bigvee_{a}^{b}(f) \bigvee_{a}^{b}(g)
$$

The constant $\frac{1}{4}$ is best possible in (12).

We also have

$$
D(f) \leq \frac{1}{2} \bigvee_{a}^{b}(f)
$$

and the constant $\frac{1}{2}$ is best possible in (13).

Using the above results we can state, for a function of bounded variation $f:[a, b] \rightarrow \mathbb{C}$, that

$$
E^{2}(f) \leq \frac{1}{2} \bigvee_{a}^{b}(f) R(f) \leq \frac{1}{2} \bigvee_{a}^{b}(f) D(f) \leq \frac{1}{4}\left[\bigvee_{a}^{b}(f)\right]^{2}
$$

In the recent paper [12] we obtained the following result that extends to complex functions the inequalities obtained in [13]:

Theorem 2. Let $f, g:[a, b] \rightarrow \mathbb{C}$ be measurable on $[a, b]$. Then 


$$
|C(f, g)| \leq\left\{\begin{array}{l}
\inf _{\gamma \in \mathbb{C}}\|g-\gamma\|_{\infty} R(f) \text { if } g \in L_{\infty}[a, b] \text { and } f \in L[a, b] \\
\frac{1}{(b-a)^{1 / q}} \inf _{\gamma \in \mathbb{C}}\|g-\gamma\|_{q} R_{p}(f), g \in L_{q}[a, b], f \in L_{p}[a, b], \\
\text { and } p, q>1 \text { with } \frac{1}{p}+\frac{1}{q}=1, \\
\frac{1}{b-a} \inf _{\gamma \in \mathbb{C}}\|g-\gamma\|_{1} R_{\infty}(f) \text { if } g \in L[a, b] \text { and } f \in L_{\infty}[a, b] .
\end{array}\right.
$$

An important corollary of this result is:

Corollary 2. Assume that $g:[a, b] \rightarrow \mathbb{C}$ is measurable on $[a, b]$ and $g \in \bar{\Delta}_{[a, b]}(\psi, \Psi)$ for some distinct complex numbers $\psi, \Psi$. Then

$$
|C(f, g)| \leq \frac{1}{2}|\Psi-\psi| R(f)
$$

if $f \in L[a, b]$.

In particular, we have

$$
D^{2}(g) \leq \frac{1}{2}|\Psi-\psi| R(g)
$$

This generalizes the following result obtained by Cheng and Sun [14] by a more complicated technique

$$
|C(f, g)| \leq \frac{1}{2}(M-m) R(f),
$$

provided $m \leq g \leq M$ for a.e. $x \in[a, b]$. The constant $\frac{1}{2}$ is best in (18) as shown by Cerone and Dragomir in [15] where a general version for Lebesgue integral and measurable spaces was also given.

Motivated by the above results, in this paper we establish other bounds for the absolute value of the Čebyšev functional when the complex-valued functions are of bounded variation. Applications to the trapezoid and mid-point inequalities are also provided.

\section{Main Results}

We have the following inequality for the complex Čebyšev functional that extends naturally the real case:

Theorem 3. If $f, g:[a, b] \rightarrow \mathbb{C}$ are Lebesgue integrable on $[a, b]$, then

$$
\max \{|C(f, g)|,|C(|f|, g)|,|C(f,|g|)|,|C(|f|,|g|)|\} \leq D(f) D(g)
$$

and

$$
\max \{|C(f, \bar{g})|,|C(|f|, \bar{g})|,|C(\bar{f},|g|)|\} \leq D(f) D(g) .
$$

Proof. As in the real case, we have Korkine's identity

$$
C(f, g):=\frac{1}{2(b-a)^{2}} \int_{a}^{b} \int_{a}^{b}(f(t)-f(s))(g(t)-g(s)) d t d s,
$$

that can be proved directly by doing the calculations in the right hand side.

By the properties of modulus, we have

$$
\begin{aligned}
|(f(t)-f(s))(g(t)-g(s))| & =|f(t)-f(s)||g(t)-g(s)| \\
& \geq\left\{\begin{array}{l}
|(|f(t)|-|f(s)|)(g(t)-g(s))|, \\
|(f(t)-f(s))(|g(t)|-|g(s)|)|, \\
|(|f(t)|-|f(s)|)(|g(t)|-|g(s)|)|
\end{array}\right.
\end{aligned}
$$


for any $t, s \in[a, b]$.

Using the properties of the integral versus the modulus, we also have

$$
\begin{aligned}
& \frac{1}{2(b-a)^{2}} \int_{a}^{b} \int_{a}^{b}|f(t)-f(s)||g(t)-g(s)| d t d s \\
& \geq \frac{1}{2(b-a)^{2}}\left\{\begin{array}{l}
\int_{a}^{b} \int_{a}^{b}|(f(t)-f(s))(g(t)-g(s))| d t d s, \\
\int_{a}^{b} \int_{a}^{b}|(|f(t)|-|f(s)|)(g(t)-g(s))| d t d s, \\
\int_{a}^{b} \int_{a}^{b}|(f(t)-f(s))(|g(t)|-|g(s)|)| d t d s, \\
\int_{a}^{b} \int_{a}^{b}|(|f(t)|-|f(s)|)(|g(t)|-|g(s)|)| d t d s,
\end{array}\right.
\end{aligned}
$$

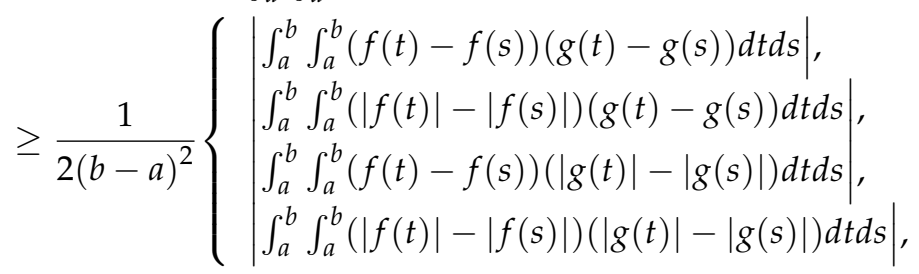

$$
\begin{aligned}
& =\left\{\begin{array}{l}
|C(f, g)|, \\
|C(|f|, g)|, \\
|C(f,|g|)|, \\
|C(|f|,|g|)| .
\end{array}\right.
\end{aligned}
$$

Using the Cauchy-Bunyakovsky-Schwarz integral inequality, we have

$$
\begin{aligned}
& \frac{1}{2(b-a)^{2}} \int_{a}^{b} \int_{a}^{b}|f(t)-f(s)||g(t)-g(s)| d t d s \\
& \leq\left[\frac{1}{2(b-a)^{2}} \int_{a}^{b} \int_{a}^{b}|f(t)-f(s)|^{2} d t d s\right]^{1 / 2} \\
& \times\left[\frac{1}{2(b-a)^{2}} \int_{a}^{b} \int_{a}^{b}|g(t)-g(s)|^{2} d t d s\right]^{1 / 2}
\end{aligned}
$$

and since

$$
\begin{aligned}
& \frac{1}{2(b-a)^{2}} \int_{a}^{b} \int_{a}^{b}|f(t)-f(s)|^{2} d t d s \\
& =\frac{1}{2(b-a)^{2}} \int_{a}^{b} \int_{a}^{b}(f(t)-f(s)) \overline{(f(t)-f(s))} d t d s \\
& =\frac{1}{2(b-a)^{2}} \int_{a}^{b} \int_{a}^{b}(f(t)-f(s))(\overline{f(t)}-\overline{f(s)}) d t d s \\
& =C(f, \bar{f})=D^{2}(f),
\end{aligned}
$$

and a similar equality for $g$, hence we obtain from (21) and (22) the desired result (19).

The inequality (20) follows from (19).

For a function of bounded variation $f:[a, b] \rightarrow \mathbb{C}$ we define the Cumulative Variation Function (CVF) of $f$ by $V_{f}:[a, b] \rightarrow[0, \infty)$ where $V_{f}(t):=\bigvee_{a}^{t}(f)$, the total variation of $f$ on the interval $[a, t]$ with $t \in[a, b]$.

It is know that the CVF is monotonic nondecreasing on $[a, b]$ and is continuous in a point $c \in[a, b]$ if and only if the generating function $f$ is continuous in that point. If $f$ is Lipschitzian with the constant $L>0$, i.e.,

$$
|f(t)-f(s)| \leq L|t-s| \text { for any } t, s \in[a, b],
$$


then $V_{f}$ is also Lipschitzian with the same constant.

Theorem 4. Let $f, g:[a, b] \rightarrow \mathbb{C}$ be Lebesgue measurable on $[a, b]$.

(i) If $f$ is of bounded variation and $g$ is Lebesgue integrable on $[a, b]$, then

$$
\max \{|C(f, g)|,|C(|f|, g)|,|C(f,|g|)|,|C(|f|,|g|)|\} \leq D\left(V_{f}\right) D(g) ;
$$

(ii) If $f$ and $g$ are of bounded variation on $[a, b]$, then

$$
\max \{|C(f, g)|,|C(|f|, g)|,|C(f,|g|)|,|C(|f|,|g|)|\} \leq C\left(V_{f}, V_{g}\right)
$$

(iii) If $f$ is Lipschitzian with the constant $L>0$ and $g$ is of bounded variation on $[a, b]$, then

$$
\max \{|C(f, g)|,|C(|f|, g)|,|C(f,|g|)|,|C(|f|,|g|)|\} \leq L C\left(\ell, V_{g}\right),
$$

where $\ell$ is the identity mapping of the interval $[a, b]$, namely $\ell(t)=t, t \in[a, b]$.

Proof. (i) If $f$ is of bounded variation then for $a \leq s<t \leq b$ we have

$$
|f(t)-f(s)| \leq \bigvee_{s}^{t}(f)=V_{f}(t)-V_{f}(s)
$$

If $a \leq t<s \leq b$ then also

$$
|f(t)-f(s)| \leq \bigvee_{t}^{s}(f)=V_{f}(s)-V_{f}(t)
$$

Therefore

$$
|f(t)-f(s)| \leq\left|V_{f}(t)-V_{f}(s)\right|
$$

for any $t, s \in[a, b]$.

Using the Cauchy-Bunyakovsky-Schwarz integral inequality, we have

$$
\begin{aligned}
& \frac{1}{2(b-a)^{2}} \int_{a}^{b} \int_{a}^{b}|f(t)-f(s)||g(t)-g(s)| d t d s \\
& \leq\left[\frac{1}{2(b-a)^{2}} \int_{a}^{b} \int_{a}^{b}|f(t)-f(s)|^{2} d t d s\right]^{1 / 2} \\
& \times\left[\frac{1}{2(b-a)^{2}} \int_{a}^{b} \int_{a}^{b}|g(t)-g(s)|^{2} d t d s\right]^{1 / 2} \\
& \leq\left[\frac{1}{2(b-a)^{2}} \int_{a}^{b} \int_{a}^{b}\left|V_{f}(t)-V_{f}(s)\right|^{2} d t d s\right]^{1 / 2} \\
& \times\left[\frac{1}{2(b-a)^{2}} \int_{a}^{b} \int_{a}^{b}|g(t)-g(s)|^{2} d t d s\right]^{1 / 2} \\
& =D\left(V_{f}\right) D(g) .
\end{aligned}
$$

Using the inequality (21) and (26) we obtain the desired result (23).

(ii) If $f$ and $g$ are of bounded variation on $[a, b]$, then for any $t, s \in[a, b]$ we have

$$
\begin{aligned}
|f(t)-f(s)||g(t)-g(s)| & \leq\left|V_{f}(t)-V_{f}(s)\right|\left|V_{g}(t)-V_{g}(s)\right| \\
& =\left(V_{f}(t)-V_{f}(s)\right)\left(V_{g}(t)-V_{g}(s)\right)
\end{aligned}
$$


since $V_{f}(\cdot)$ and $V_{g}(\cdot)$ are monotonic nondecreasing on $[a, b]$.

Then

$$
\begin{aligned}
& \frac{1}{2(b-a)^{2}} \int_{a}^{b} \int_{a}^{b}|f(t)-f(s)||g(t)-g(s)| d t d s \\
& \leq \frac{1}{2(b-a)^{2}} \int_{a}^{b} \int_{a}^{b}\left(V_{f}(t)-V_{f}(s)\right)\left(V_{g}(t)-V_{g}(s)\right) d t d s \\
& =C\left(V_{f}, V_{g}\right) .
\end{aligned}
$$

Using the inequality (21) and (27) we obtain the desired result (24).

(iii) If $f$ is Lipschitzian with the constant $L>0$ and $g$ is of bounded variation on $[a, b]$, then

$$
\begin{aligned}
|f(t)-f(s)||g(t)-g(s)| & \leq L|t-s|\left|V_{g}(t)-V_{g}(s)\right| \\
& =L(t-s)\left(V_{g}(t)-V_{g}(s)\right)
\end{aligned}
$$

since $V_{f}(\cdot)$ is monotonic nondecreasing on $[a, b]$.

Then

$$
\begin{aligned}
& \frac{1}{2(b-a)^{2}} \int_{a}^{b} \int_{a}^{b}|f(t)-f(s)||g(t)-g(s)| d t d s \\
& \leq \frac{1}{2(b-a)^{2}} L \int_{a}^{b} \int_{a}^{b}(t-s)\left(V_{g}(t)-V_{g}(s)\right) d t d s \\
& =L C\left(V_{f}, V_{g}\right) .
\end{aligned}
$$

Using the inequality (21) and (28) we obtain the desired result (25).

In 1970, A. M. Ostrowski [16] proved among other things the following result, which is somehow a mixture of the Čebyšev and Grüss results

$$
|C(f, g)| \leq \frac{1}{8}(b-a)(M-m)\left\|g^{\prime}\right\|_{\infty}
$$

provided $f$ is Lebesgue integrable on $[a, b]$ and satisfying (2) while $g:[a, b] \rightarrow \mathbb{R}$ is absolutely continuous and $g^{\prime} \in L_{\infty}[a, b]$. Here the constant $\frac{1}{8}$ is also sharp.

The following lemma for real-valued functions holds [17].

Lemma 1. Let $h:[a, b] \rightarrow \mathbb{R}$ be an integrable function on $[a, b]$ such that

$$
-\infty<\gamma \leq h(x) \leq \Gamma<\infty \text { for a.e. } x \text { on }[a, b] \text {. }
$$

Then we have the inequality

$$
\begin{aligned}
& \frac{1}{b-a} \int_{a}^{b}\left|\int_{a}^{x} h(t) d t-\frac{x-a}{b-a} \int_{a}^{b} h(u) d u\right| d x \\
& \leq \frac{1}{2}\left(\frac{1}{b-a} \int_{a}^{b} h(u) d u-\gamma\right)\left(\Gamma-\frac{1}{b-a} \int_{a}^{b} h(u) d u\right) \frac{b-a}{\Gamma-\gamma} \\
& \leq \frac{1}{8}(\Gamma-\gamma)(b-a) .
\end{aligned}
$$

The constants $\frac{1}{2}$ and $\frac{1}{8}$ are sharp in the sense that they cannot be replaced by smaller constants.

When one function is complex-valued, we can state the following refinement and extension of Ostrowski's inequality (29). This extends the corresponding result from [17] in which both functions are real-valued. 
Theorem 5. Let $f:[a, b] \rightarrow \mathbb{R}$ be measurable and such that there exist the constants $m$, $M \in \mathbb{R}$ with

$$
-\infty<m \leq f(x) \leq M<\infty \text { for a.e. } x \text { on }[a, b]
$$

If $g:[a, b] \rightarrow \mathbb{C}$ is absolutely continuous on $[a, b]$ with $g^{\prime} \in L_{\infty}[a, b]$ then we have the inequality

$$
\begin{aligned}
|C(f, g)| & \leq \frac{1}{2}\left\|g^{\prime}\right\|_{\infty} \frac{\left(\frac{1}{b-a} \int_{a}^{b} f(x) d x-m\right)\left(M-\frac{1}{b-a} \int_{a}^{b} f(x) d x\right)}{M-m}(b-a) \\
& \leq \frac{1}{8}(b-a)(M-m)\left\|g^{\prime}\right\|_{\infty} .
\end{aligned}
$$

The constants $\frac{1}{2}$ and $\frac{1}{8}$ are sharp in the above sense.

Proof. Integrating by parts, we have

$$
\begin{aligned}
& \frac{1}{b-a} \int_{a}^{b}\left(\int_{a}^{x} f(t) d t-\frac{x-a}{b-a} \int_{a}^{b} f(u) d u\right) g^{\prime}(x) d x \\
& =\frac{1}{b-a}\left[\left.\left(\int_{a}^{x} f(t) d t-\frac{x-a}{b-a} \int_{a}^{b} f(u) d u\right) g(x)\right|_{a} ^{b}\right. \\
& \left.-\int_{a}^{b} g(x)\left[f(x)-\frac{1}{b-a} \int_{a}^{b} f(u) d u\right] d x\right] \\
& =-\frac{1}{b-a} \int_{a}^{b} g(x) f(x) d x+\frac{1}{b-a} \int_{a}^{b} g(x) d x \cdot \frac{1}{b-a} \int_{a}^{b} f(x) d x \\
& =-C(f, g) .
\end{aligned}
$$

Taking the modulus, we have

$$
\begin{aligned}
|C(f, g)| & \leq \frac{1}{b-a} \int_{a}^{b}\left|\int_{a}^{x} f(t) d t-\frac{x-a}{b-a} \int_{a}^{b} f(u) d u\right|\left|g^{\prime}(x)\right| d x \\
& \leq\left\|g^{\prime}\right\|_{\infty} \frac{1}{b-a} \int_{a}^{b}\left|\int_{a}^{x} f(t) d t-\frac{x-a}{b-a} \int_{a}^{b} f(u) d u\right| d x \text { (by Lemma 1) } \\
& \leq \frac{1}{2}\left\|g^{\prime}\right\|_{\infty} \frac{\left(\frac{1}{b-a} \int_{a}^{b} f(x) d x-m\right)\left(M-\frac{1}{b-a} \int_{a}^{b} f(x) d x\right)}{M-m}(b-a) .
\end{aligned}
$$

The sharpness of the constants follows from the real-valued case outlined in [17].

\section{Some Examples}

Assume that the function $f:[a, b] \rightarrow \mathbb{C}$ is of bounded variation on $[a, b]$. Since the function $V_{f}$ is monotonic nondecreasing on $[a, b]$, then $0 \leq V_{f}(t) \leq \bigvee_{a}^{b}(f)$ for any $t \in[a, b]$, and by (8) we have $D\left(V_{f}\right) \leq \frac{1}{2} \bigvee_{a}^{b}(f)$. Using (23) we have

$$
\max \{|C(f, g)|,|C(|f|, g)|,|C(f,|g|)|,|C(|f|,|g|)|\} \leq \frac{1}{2} \bigvee_{a}^{b}(f) D(g)
$$

for any $g$ Lebesgue integrable function on $[a, b]$.

If $g \in \bar{\Delta}_{[a, b]}(\psi, \Psi)$ for some distinct complex numbers $\psi, \Psi$, then by (34) we have

$$
\max \{|C(f, g)|,|C(|f|, g)|,|C(f,|g|)|,|C(|f|,|g|)|\} \leq \frac{1}{4}|\Psi-\psi| \bigvee_{a}^{b}(f)
$$


If $f$ and $g$ are of bounded variation on $[a, b]$, then $V_{f}$ and $V_{g}$ are monotonic nondecreasing on $[a, b]$ and by (12) we have

$$
\left|C\left(V_{f}, V_{g}\right)\right| \leq \frac{1}{2} \bigvee_{a}^{b}(f) R\left(V_{g}\right) \leq \frac{1}{2} \bigvee_{a}^{b}(f) D\left(V_{g}\right) \leq \frac{1}{4} \bigvee_{a}^{b}(f) \bigvee_{a}^{b}(g)
$$

Using the inequality (24) we then have

$$
\begin{aligned}
& \max \{|C(f, g)|,|C(|f|, g)|,|C(f,|g|)|,|C(|f|,|g|)|\} \\
& \leq \frac{1}{2} \bigvee_{a}^{b}(f) R\left(V_{g}\right) \leq \frac{1}{2} \bigvee_{a}^{b}(f) D\left(V_{g}\right) \leq \frac{1}{4} \bigvee_{a}^{b}(f) \bigvee_{a}^{b}(g)
\end{aligned}
$$

If $f$ and $g$ are of bounded variation on $[a, b]$, then by (15) we have

$$
\left|C\left(V_{f}, V_{g}\right)\right| \leq\left\{\begin{array}{l}
\inf _{\gamma \in \mathbb{C}}\left\|V_{g}-\gamma\right\|_{\infty} R\left(V_{f}\right), \\
\frac{1}{(b-a)^{1 / q}} \inf _{\gamma \in \mathbb{C}}\left\|V_{g}-\gamma\right\|_{q} R_{p}\left(V_{f}\right), \\
\text { where } p, q>1 \text { with } \frac{1}{p}+\frac{1}{q}=1, \\
\frac{1}{b-a} \inf _{\gamma \in \mathbb{C}}\left\|V_{g}-\gamma\right\|_{1} R_{\infty}\left(V_{f}\right) .
\end{array}\right.
$$

Using the inequality (24) we then have

$$
\begin{aligned}
& \max \{|C(f, g)|,|C(|f|, g)|,|C(f,|g|)|,|C(|f|,|g|)|\} \\
& \leq\left\{\begin{array}{l}
\inf _{\gamma \in \mathbb{C}}\left\|V_{g}-\gamma\right\|_{\infty} R\left(V_{f}\right), \\
\frac{1}{(b-a)^{1 / q}} \inf _{\gamma \in \mathbb{C}}\left\|V_{g}-\gamma\right\|_{q} R_{p}\left(V_{f}\right), \\
\text { where } p, q>1 \text { with } \frac{1}{p}+\frac{1}{q}=1, \\
\frac{1}{b-a} \inf _{\gamma \in \mathbb{C}}\left\|V_{g}-\gamma\right\|_{1} R_{\infty}\left(V_{f}\right) .
\end{array}\right.
\end{aligned}
$$

From (39) we obtain in particular

$$
\begin{aligned}
& \max \{|C(f, g)|,|C(|f|, g)|,|C(f,|g|)|,|C(|f|,|g|)|\} \\
& \leq\left\|V_{g}-\frac{1}{2} \bigvee_{a}^{b}(g)\right\|_{\infty} R\left(V_{f}\right) \leq \frac{1}{2} \bigvee_{a}^{b}(g) R\left(V_{f}\right)
\end{aligned}
$$

Now, we observe that for $f=\ell$, where $\ell$ is the identity mapping of the interval $[a, b]$, namely $\ell(t)=t, t \in[a, b]$, we have

$$
R(\ell)=\frac{1}{b-a} \int_{a}^{b}\left|t-\frac{a+b}{2}\right| d t=\frac{1}{4}(b-a) .
$$

Then we have by (18) that

$$
\left|C\left(\ell, V_{g}\right)\right| \leq \frac{1}{8}(b-a) \bigvee_{a}^{b}(g) .
$$

Therefore, if $f$ is Lipschitzian with the constant $L>0$ and $g$ is of bounded variation on $[a, b]$, then by (25) and (41) we have 


$$
\max \{|C(f, g)|,|C(|f|, g)|,|C(f,|g|)|,|C(|f|,|g|)|\} \leq \frac{1}{8}(b-a) L \bigvee_{a}^{b}(g)
$$

From (33) for $f=V_{h}$ and $g=\ell$ we have $m=0$ and $M=\bigvee_{a}^{b}(h)$ and

$$
\begin{aligned}
\left|C\left(\ell, V_{h}\right)\right| & \leq \frac{1}{2}\left(1-\frac{1}{(b-a) \bigvee_{a}^{b}(h)} \int_{a}^{b} V_{h}(t) d t\right) \int_{a}^{b} V_{h}(t) d t \\
& \leq \frac{1}{8}(b-a) \bigvee_{a}^{b}(h),
\end{aligned}
$$

provided that $h:[a, b] \rightarrow \mathbb{C}$ is of bounded variation on $[a, b]$.

Therefore, if $f$ is Lipschitzian with the constant $L>0$ and $g$ is of bounded variation on $[a, b]$, then by (25) and (43) we have

$$
\begin{aligned}
& \max \{|C(f, g)|,|C(|f|, g)|,|C(f,|g|)|,|C(|f|,|g|)|\} \\
& \leq \frac{1}{2} L\left(1-\frac{1}{(b-a) \bigvee_{a}^{b}(g)} \int_{a}^{b} V_{g}(t) d t\right) \int_{a}^{b} V_{g}(t) d t \leq \frac{1}{8}(b-a) L \bigvee_{a}^{b}(g) .
\end{aligned}
$$

This is an improvement of the inequality (42) above.

It is known that, if $h:[a, b] \rightarrow \mathbb{C}$ is absolutely continuous on $[a, b]$, then [18] (Theorem 16), $V_{h}$ is absolutely continuous and $V_{h}(x)=\int_{a}^{x}\left|h^{\prime}(t)\right| d t$ for any $x \in[a, b]$. Moreover, $V_{h}^{\prime}(x)=\left|h^{\prime}(x)\right|$ for a.e. $x \in[a, b]$.

Assume that $g:[a, b] \rightarrow \mathbb{C}$ is absolutely continuous with $g^{\prime} \in L_{\infty}[a, b]$ and $f:[a, b] \rightarrow$ $\mathbb{C}$ is of bounded variation. From (33) for $V_{f}, V_{g}$ we have

$$
\begin{aligned}
\left|C\left(V_{f}, V_{g}\right)\right| & \leq \frac{1}{2}\left\|g^{\prime}\right\|_{\infty}\left(1-\frac{1}{(b-a) \bigvee_{a}^{b}(g)} \int_{a}^{b} V_{g}(t) d t\right) \int_{a}^{b} V_{g}(t) d t \\
& \leq \frac{1}{8}(b-a) \bigvee_{a}^{b}(f)\left\|g^{\prime}\right\|_{\infty} .
\end{aligned}
$$

Using (24) we then have

$$
\begin{aligned}
& \max \{|C(f, g)|,|C(|f|, g)|,|C(f,|g|)|,|C(|f|,|g|)|\} \\
& \leq \frac{1}{2}\left\|g^{\prime}\right\|_{\infty}\left(1-\frac{1}{(b-a) \bigvee_{a}^{b}(g)} \int_{a}^{b} V_{g}(t) d t\right) \int_{a}^{b} V_{g}(t) d t \\
& \leq \frac{1}{8}(b-a) \bigvee_{a}^{b}(f)\left\|g^{\prime}\right\|_{\infty^{\prime}}
\end{aligned}
$$

provided that $g:[a, b] \rightarrow \mathbb{C}$ is absolutely continuous with $g^{\prime} \in L_{\infty}[a, b]$ and $f:[a, b] \rightarrow \mathbb{C}$ is of bounded variation on $[a, b]$. 


\section{Applications to Trapezoid Inequality}

Let $h:[a, b] \rightarrow \mathbb{C}$ be an absolutely continuous function on $[a, b]$. Then we have the trapezoid equality

$$
\frac{h(a)+h(b)}{2}-\frac{1}{b-a} \int_{a}^{b} h(t) d t=\frac{1}{b-a} \int_{a}^{b}\left(t-\frac{a+b}{2}\right)\left(h^{\prime}(t)-\delta\right) d t
$$

for any $\delta \in \mathbb{C}$. This is obvious integrating by parts in the right hand side of the equality.

Using the inequality (12) for $f=h^{\prime}-\delta$ and $g=\ell-\frac{a+b}{2}$ we obtain

$$
\frac{1}{b-a}\left|\int_{a}^{b}\left(t-\frac{a+b}{2}\right)\left(h^{\prime}(t)-\delta\right) d t\right| \leq \frac{1}{2} \bigvee_{a}^{b}\left(h^{\prime}-\delta\right) R\left(\ell-\frac{a+b}{2}\right)
$$

Since

$$
\begin{aligned}
R\left(\ell-\frac{a+b}{2}\right) & =\frac{1}{b-a} \int_{a}^{b}\left|t-\frac{a+b}{2}-\frac{1}{b-a} \int_{a}^{b}\left(s-\frac{a+b}{2}\right) d s\right| \\
& =\frac{1}{b-a} \int_{a}^{b}\left|t-\frac{a+b}{2}\right| d t=\frac{1}{4}(b-a),
\end{aligned}
$$

then by (47) and (48) we obtain

$$
\left|\frac{h(a)+h(b)}{2}-\frac{1}{b-a} \int_{a}^{b} h(t) d t\right| \leq \frac{1}{8}(b-a) \bigvee_{a}^{b}\left(h^{\prime}-\delta\right)
$$

provided that $h^{\prime}$ is of bounded variation and $\delta \in \mathbb{C}$.

If

$$
\bigvee_{a}^{b}\left(h^{\prime}-\frac{\Psi+\psi}{2}\right) \leq \frac{1}{2}(b-a)|\Psi-\psi|
$$

for some complex numbers $\psi, \Psi \in \mathbb{C}$, then by (49) we obtain

$$
\left|\frac{h(a)+h(b)}{2}-\frac{1}{b-a} \int_{a}^{b} h(t) d t\right| \leq \frac{1}{16}(b-a)^{2}|\Psi-\psi|,
$$

provided $h$ satisfies the condition (50). We observe that, a sufficient condition for the condition (50) to hold is that $h$ is twice differentiable on $(a, b)$ and $h^{\prime \prime} \in \bar{\Delta}_{[a, b]}(\psi, \Psi)$.

Using the inequality (12) for $f=\ell-\frac{a+b}{2}$ and $g=h^{\prime}-\delta$ we obtain

$$
\frac{1}{b-a}\left|\int_{a}^{b}\left(t-\frac{a+b}{2}\right)\left(h^{\prime}(t)-\delta\right) d t\right| \leq \frac{1}{2} \bigvee_{a}^{b}\left(\ell-\frac{a+b}{2}\right) R\left(h^{\prime}-\delta\right)
$$

Since

$$
\bigvee_{a}^{b}\left(\ell-\frac{a+b}{2}\right)=b-a
$$

and

$$
R\left(h^{\prime}-\delta\right)=\frac{1}{b-a} \int_{a}^{b}\left|h^{\prime}(t)-\frac{h(b)-h(a)}{b-a}\right| d t,
$$

then by (52) and (47) we obtain

$$
\left|\frac{h(a)+h(b)}{2}-\frac{1}{b-a} \int_{a}^{b} h(t) d t\right| \leq \frac{1}{2} \int_{a}^{b}\left|h^{\prime}(t)-\frac{h(b)-h(a)}{b-a}\right| d t
$$

provided $h:[a, b] \rightarrow \mathbb{C}$ is an absolutely continuous function on $[a, b]$.

If we use the inequality (16) for $f=h^{\prime}$ with $h^{\prime} \in \bar{\Delta}_{[a, b]}(\phi, \Phi)$ for some distinct complex 
numbers $\phi, \Phi$ and $g=\ell-\frac{a+b}{2}$, then

$$
\begin{aligned}
\frac{1}{b-a}\left|\int_{a}^{b}\left(t-\frac{a+b}{2}\right) h^{\prime}(t) d t\right| & \leq \frac{1}{2}|\Phi-\phi| R\left(\ell-\frac{a+b}{2}\right) \\
& =\frac{1}{8}(b-a)|\Phi-\phi| .
\end{aligned}
$$

Using (47) we then obtain

$$
\left|\frac{h(a)+h(b)}{2}-\frac{1}{b-a} \int_{a}^{b} h(t) d t\right| \leq \frac{1}{8}(b-a)|\Phi-\phi|
$$

provided $h:[a, b] \rightarrow \mathbb{C}$ is an absolutely continuous function on $[a, b]$ and there exist the distinct complex numbers $\phi, \Phi$ such that $h^{\prime} \in \bar{\Delta}_{[a, b]}(\phi, \Phi)$.

For $g=\ell-\frac{a+b}{2}$ we have

$$
V_{g}(t)=\bigvee_{a}^{t}\left(\ell-\frac{a+b}{2}\right)=t-a, t \in[a, b]
$$

If we use the inequality (44) for $f=h^{\prime}$ and $g=\ell-\frac{a+b}{2}$ we obtain

$$
\begin{aligned}
& \frac{1}{b-a}\left|\int_{a}^{b}\left(t-\frac{a+b}{2}\right) h^{\prime}(t) d t\right| \\
& \leq \frac{1}{2} K\left(1-\frac{1}{(b-a) \bigvee_{a}^{b}(g)} \int_{a}^{b}(t-a) d t\right) \int_{a}^{b}(t-a) d t
\end{aligned}
$$

namely

$$
\frac{1}{b-a}\left|\int_{a}^{b}\left(t-\frac{a+b}{2}\right) h^{\prime}(t) d t\right| \leq \frac{1}{8}(b-a)^{2} K,
$$

provided $h^{\prime}$ is Lipschitzian with the constant $K>0$.

If we use (47) and (56), we obtain

$$
\left|\frac{h(a)+h(b)}{2}-\frac{1}{b-a} \int_{a}^{b} h(t) d t\right| \leq \frac{1}{8}(b-a)^{2} K
$$

provided $h^{\prime}$ is Lipschitzian with the constant $K>0$.

\section{Applications to Mid-Point Inequality}

Let $h:[a, b] \rightarrow \mathbb{C}$ be an absolutely continuous function on $[a, b]$. Then we have the mid-point equality

$$
h\left(\frac{a+b}{2}\right)-\frac{1}{b-a} \int_{a}^{b} h(t) d t=\frac{1}{b-a} \int_{a}^{b} p(t)\left(h^{\prime}(t)-\delta\right) d t
$$

for any $\delta \in \mathbb{C}$, where the kernel $p:[a, b] \rightarrow \mathbb{R}$ is given by

$$
p(t):=\left\{\begin{array}{l}
t-a \text { if } t \in\left[a, \frac{a+b}{2}\right], \\
t-b \text { if } t \in\left(\frac{a+b}{2}, b\right] .
\end{array}\right.
$$

This is obvious integrating by parts in the right hand side of the equality.

Using the inequality (12) for $f=h^{\prime}-\delta$ and $g=p$ we obtain 


$$
\frac{1}{b-a}\left|\int_{a}^{b} p(t)\left(h^{\prime}(t)-\delta\right) d t\right| \leq \frac{1}{2} \bigvee_{a}^{b}\left(h^{\prime}-\delta\right) R(p) .
$$

Since

$$
R(p)=\frac{1}{b-a} \int_{a}^{b}|p(t)| d t=\frac{1}{4}(b-a),
$$

then by (57) and (59) we obtain

$$
\left|h\left(\frac{a+b}{2}\right)-\frac{1}{b-a} \int_{a}^{b} h(t) d t\right| \leq \frac{1}{8}(b-a) \bigvee_{a}^{b}\left(h^{\prime}-\delta\right)
$$

provided that $h^{\prime}$ is of bounded variation and $\delta \in \mathbb{C}$.

If $h$ satisfies the condition (50) for some complex numbers $\psi, \Psi \in \mathbb{C}$, then by (60) we obtain

$$
\left|h\left(\frac{a+b}{2}\right)-\frac{1}{b-a} \int_{a}^{b} h(t) d t\right| \leq \frac{1}{16}(b-a)^{2}|\Psi-\psi|
$$

provided $h$ satisfies the condition (50). We observe that, a sufficient condition for the condition (50) to hold is that $h$ is twice differentiable on $(a, b)$ and $h^{\prime \prime} \in \bar{\Delta}_{[a, b]}(\psi, \Psi)$.

Using the inequality (12) for $f=p$ and $g=h^{\prime}-\delta$ we obtain

$$
\frac{1}{b-a}\left|\int_{a}^{b} p(t)\left(h^{\prime}(t)-\delta\right) d t\right| \leq \frac{1}{2} \bigvee_{a}^{b}(p) R\left(h^{\prime}-\delta\right) .
$$

Since $\bigvee_{a}^{b}(p)=b-a$ and

$$
R\left(h^{\prime}-\delta\right)=\frac{1}{b-a} \int_{a}^{b}\left|h^{\prime}(t)-\frac{h(b)-h(a)}{b-a}\right| d t,
$$

then by (57) and (62) we obtain

$$
\left|h\left(\frac{a+b}{2}\right)-\frac{1}{b-a} \int_{a}^{b} h(t) d t\right| \leq \frac{1}{2} \int_{a}^{b}\left|h^{\prime}(t)-\frac{h(b)-h(a)}{b-a}\right| d t
$$

provided $h:[a, b] \rightarrow \mathbb{C}$ is an absolutely continuous function on $[a, b]$.

If we use the inequality (16) for $f=h^{\prime}$ with $h^{\prime} \in \bar{\Delta}_{[a, b]}(\phi, \Phi)$ for some distinct complex numbers $\phi, \Phi$ and $g=p$, then

$$
\frac{1}{b-a}\left|\int_{a}^{b} p(t) h^{\prime}(t) d t\right| \leq \frac{1}{2}|\Phi-\phi| R(p)=\frac{1}{8}(b-a)|\Phi-\phi| .
$$

By (57) and (64) we obtain

$$
\left|h\left(\frac{a+b}{2}\right)-\frac{1}{b-a} \int_{a}^{b} h(t) d t\right| \leq \frac{1}{8}(b-a)|\Phi-\phi|,
$$

provided that $h:[a, b] \rightarrow \mathbb{C}$ is an absolutely continuous function on $[a, b]$ and $h^{\prime} \in$ $\bar{\Delta}_{[a, b]}(\phi, \Phi)$ for some distinct complex numbers $\phi, \Phi$.

The kernel $p$ defined by (58) is of bounded variation and we have for $t \in\left[a, \frac{a+b}{2}\right]$ that $V_{p}(t)=\bigvee_{a}^{t}(p)=t-a$ and for $t \in\left(\frac{a+b}{2}, b\right]$

$$
V_{p}(t)=\bigvee_{a}^{t}(p)=\bigvee_{a}^{\frac{a+b}{2}}(p)+\bigvee_{\frac{a+b}{2}}^{t}(p)=t-a,
$$


therefore, for any $t \in[a, b]$ we have $V_{p}(t)=t-a$. We observe that, this is an example of a function of bounded variation for the CVF is differentiable for every $t \in[a, b]$.

Now, if we take $g=p$ and $f=h^{\prime}-\delta$ and use the inequality (24), then we obtain

$$
\begin{aligned}
& \left|h\left(\frac{a+b}{2}\right)-\frac{1}{b-a} \int_{a}^{b} h(t) d t\right| \\
& \leq \frac{1}{b-a} \int_{a}^{b} V_{h^{\prime}-\delta}(t)(t-a) d t-\frac{1}{2} \int_{a}^{b} V_{h^{\prime}-\delta}(t) d t .
\end{aligned}
$$

Using the inequality (33) we also have

$$
\begin{aligned}
& \left|C\left(V_{h^{\prime}-\delta}, \ell-a\right)\right| \\
& \leq \frac{1}{2} \frac{\left(\frac{1}{b-a} \int_{a}^{b} V_{h^{\prime}-\delta}(x) d x\right)\left(\bigvee_{a}^{b}\left(h^{\prime}-\delta\right)-\frac{1}{b-a} \int_{a}^{b} V_{h^{\prime}-\delta}(x) d x\right)}{\bigvee_{a}^{b}\left(h^{\prime}-\delta\right)}(b-a) \\
& \leq \frac{1}{8}(b-a) \bigvee_{a}^{b}\left(h^{\prime}-\delta\right) .
\end{aligned}
$$

Then by (57) and (66) we obtain

$$
\begin{aligned}
& \left|h\left(\frac{a+b}{2}\right)-\frac{1}{b-a} \int_{a}^{b} h(t) d t\right| \\
& \leq \frac{1}{2}\left(1-\frac{1}{(b-a) \bigvee_{a}^{b}\left(h^{\prime}-\delta\right)} \int_{a}^{b} V_{h^{\prime}-\delta}(x) d x\right) \int_{a}^{b} V_{h^{\prime}-\delta}(x) d x \\
& \leq \frac{1}{8}(b-a) \bigvee_{a}^{b}\left(h^{\prime}-\delta\right)
\end{aligned}
$$

for any $\delta \in \mathbb{C}$, where $h:[a, b] \rightarrow \mathbb{C}$ is an absolutely continuous function on $[a, b]$ and the derivative is of bounded variation. This is a refinement of (60).

\section{Conclusions}

In this paper, we provide several bounds for the modulus of the complex Čebyšev functional

$$
C(f, g):=\frac{1}{b-a} \int_{a}^{b} f(t) g(t) d t-\frac{1}{b-a} \int_{a}^{b} f(t) d t \int_{a}^{b} g(t) d t
$$

under various assumptions for the integrable functions $f, g:[a, b] \rightarrow \mathbb{C}$. We show among other things that, if $f$ and $g$ are of bounded variation on $[a, b]$, then

$$
\max \{|C(f, g)|,|C(|f|, g)|,|C(f,|g|)|,|C(|f|,|g|)|\} \leq C\left(V_{f}, V_{g}\right),
$$

where the cumulative variation function $V_{f}:[a, b] \rightarrow[0, \infty)$ is defined by $V_{f}(t):=\bigvee_{a}^{t}(f)$. Applications to the trapezoid and mid-point inequalities are also provided.

Funding: This research received no external funding.

Institutional Review Board Statement: Not applicable.

Informed Consent Statement: Not applicable.

Data Availability Statement: Not applicable.

Conflicts of Interest: The author declares no conflict of interest. 


\section{References}

1. Grüss, G. Über das maximum des absoluten Betrages von $\frac{1}{b-a} \int_{a}^{b} f(x) g(x) d x-\frac{1}{(b-a)^{2}} \int_{a}^{b} f(x) d x \cdot \int_{a}^{b} g(x) d x$. Math. Z. 1934, 39, 215-226. [CrossRef]

2. Cerone, P.; Dragomir, S.S. Some new Ostrowski-type bounds for the Čebyšev functional and applications. J. Math. Inequal. 2014, 8, 159-170. [CrossRef]

3. Chebyshev, P.L. Sur les expressions approximatives des intè grals dèfinis par les outres prises entre les même limites. Proc. Math. Soc. Charkov 1882, 2, 93-98.

4. Dragomir, S.S. A generalization of Grüss's inequality in inner product spaces and applications. J. Math. Anal. Appl. 1999, 237, 74-82. [CrossRef]

5. Dragomir, S.S. Some integral inequalities of Gruss type. Indian J. Pure Appl. Math. 2000, 31, 397-415. [CrossRef]

6. Iordanescu, R.; Nichita, F.F.; Pasarescu, O. Unification Theories: Means and Generalized Euler Formulas. Axioms 2020, 9 , 144. [CrossRef]

7. Lupaş, A. The best constant in an integral inequality. Mathematica (Cluj) 1973, 15, $219-222$.

8. Dragomir, S.S. Some Grüss type inequalities in inner product spaces. J. Inequal. Pure Appl. Math. $2003,4,42$.

9. Dragomir, S.S.; Moslehian, M.S.; Cho, Y.J. Some reverses of the Cauchy-Schwarz inequality for complex functions of self-adjoint operators in Hilbert spaces. Math. Inequal. Appl. 2014, 17, 1365-1373; reprinted in RGMIA Res. Rep. Coll. 2011, 14, 84. [CrossRef]

10. Dragomir, S.S. New Grüss' type inequalities for functions of bounded variation and applications. Appl. Math. Lett. 2012, 25, 1475-1479. [CrossRef]

11. Dragomir, S.S. Bounding the Cebysev functional for functions of bounded variation and applications. RGMIA Res. Rep. Coll. 2011, 14, 5 .

12. Dragomir, S.S. Integral Grüss' type inequalities for complex-valued functions. RGMIA Res. Rep. Coll. 2017, $20,13$.

13. Cerone, P.; Dragomir, S.S. New bounds for the Čebyš ev functional. Appl. Math. Lett. 2005, 18, 603-611. [CrossRef]

14. Cheng, X.-L.; Sun, J. Note on the perturbed trapezoid inequality. J. Inequal. Pure Appl. Math. 2002, 3, 29.

15. Cerone, P.; Dragomir, S.S. A refinement of the Grüss inequality and applications. Tamkang J. Math. 2007, 38, 37-49; reprinted in RGMIA Res. Rep. Coll. 2002, 5, 14. [CrossRef]

16. Ostrowski, A.M. On an integral inequality. Aequat. Math. 1970, 4, 358-373. [CrossRef]

17. Dragomir, S.S. A refinement of Ostrowski's inequality for the Čebyšev functional and applications. Analysis (Munich) 2003, 23, 287-297. [CrossRef]

18. Heil, C.H. Real Analysis Lecture Notes, Absolutely Continuous and Singular Functions. Available online: https://people.math. ethz.ch/ fdalio/old/VorlesungenMassundIntegralFS16/ACfunctions.pdf (accessed on 26 May 2021 ). 